\title{
Four-Point Resistance of Individual Single-Wall Carbon Nanotubes
}

\author{
B. Gao, ${ }^{1}$ Y. F. Chen, ${ }^{2}$ M. S. Fuhrer, ${ }^{2}$ D. C. Glattli, ${ }^{1,3}$ and A. Bachtold ${ }^{1,4, *}$ \\ ${ }^{1}$ Laboratoire Pierre Aigrain, Ecole Normale Supérieure, 75231 Paris 05, France \\ ${ }^{2}$ Department of Physics and Center for Superconductivity Research, University of Maryland, College Park, Maryland 20742, USA \\ ${ }^{3}$ SPEC, CEA Saclay, F-91191 Gif-sur-Yvette, France \\ ${ }^{4}$ CNM-CSIC, Campus Universitat Autonoma de Barcelona, E-08193 Bellaterra, Spain
}

(Received 19 April 2005; published 31 October 2005)

\begin{abstract}
We have studied the resistance of single-wall carbon nanotubes measured in a four-point configuration with noninvasive voltage electrodes. The voltage drop is detected using multiwalled carbon nanotubes while the current is injected through nanofabricated Au electrodes. The resistance at room temperature is shown to be linear with the length as expected for a classical resistor. This changes at cryogenic temperature; the four-point resistance then depends on the resistance at the Au-tube interfaces and can even become negative due to quantum-interference effects.
\end{abstract}

DOI: $10.1103 /$ PhysRevLett.95.196802

Transport measurements are a powerful technique to investigate electronic properties of molecular systems [1]. Most often, individual molecular systems are electrically attached to two nanofabricated electrodes. However, such two-point experiments do not allow the determination of the intrinsic resistance that results from scattering processes involving, e.g., phonons or disorder. Indeed, the resistance is mainly dominated by poorly defined contacts that lie in series. A solution to eliminate the contribution of contacts has been found with scanning probe microscopy techniques [2-6], which enable the measurement of resistance variations along long systems such as nanotubes, but these techniques have only been applied at room temperature. The standard method to determine the intrinsic resistivity of macroscopic systems is the four-point measurement. The application of this technique to molecular systems is challenging, however, since the electrodes used so far have been invasive. For example, nanofabricated electrodes were shown to divide nanotubes into multiple quantum dots [7].

We report four-point resistance measurements on singlewall carbon nanotubes (SWNTs) using multiwalled carbon nanotubes (MWNTs) as noninvasive voltage electrodes [Fig. 1(a)]. We find that SWNTs are remarkably good one-dimensional conductors with resistances as low as $1.5 \mathrm{k} \Omega$ for a $95 \mathrm{~nm}$ long section. The nanotube resistance is shown to linearly increase with length at room temperature, in agreement with Ohm's law. At low temperature, however, the resistance can become negative and the amplitude then depends on the resistance at the Au-SWNT interfaces. In this regime, four-point measurements can be described by the Landauer-Büttiker formalism taking into account quantum-interference effects.

Before discussing these measurements, we briefly review the basic physics of four-point measurements [Fig. 1(b)]. In the diffusive incoherent limit, the four-point resistance $R_{4 \mathrm{pt}}$ of SWNTs, characterized by 4 conducting channels, is given by
PACS numbers: 73.63.Fg, 72.15.Lh, 72.80.Rj, 73.23.-b

$$
R_{4 \mathrm{pt}}=\frac{h}{4 e^{2}} \frac{L}{l_{e}}
$$

with $l_{e}$ the elastic mean-free path and $L$ the separation between the voltage electrodes. Equation (1) describes the intrinsic resistance generated by the electronic backscattering along the nanotube.

Equation (1) requires the transmission to the voltage electrodes to be weak to suppress an additional resistance contribution that results from electrons entering the voltage electrodes. Such electrons are replaced by electrons that scatter into either direction of the tube, enhancing the resistance [8].

By introducing quantum-interference effects, four-point measurements can give striking results such as negative $R_{4 \mathrm{pt}}$. This is best seen using the Büttiker formula [9-11], which is convenient for the description of multiterminal conductors. The current $I_{\alpha}$ in each electrode is related to

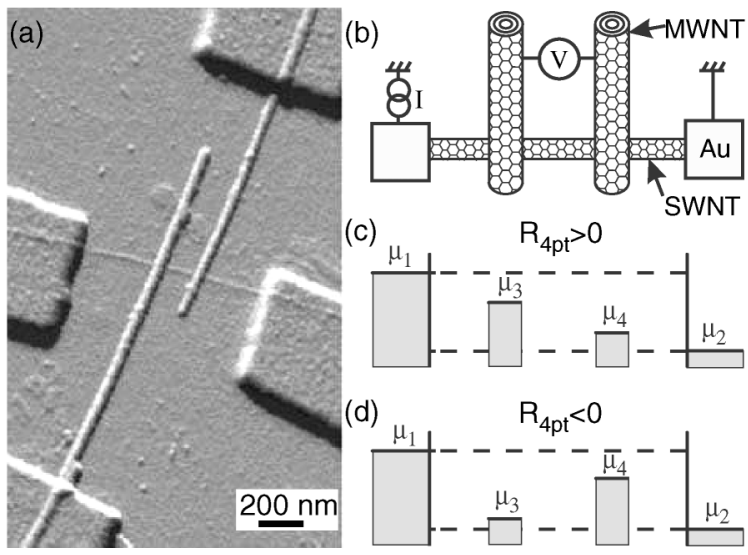

FIG. 1. (a) Atomic force microscopy image of a SWNT contacted by 2 MWNTs and 2 Au electrodes. (b) Schematic of the $R_{4 \mathrm{pt}}$ measurement. (c), (d) Levels of the electrochemical potential for the 4 electrodes that give a positive $R_{4 \mathrm{pt}}$ in (c) and a negative $R_{4 \mathrm{pt}}$ in (d). 
the electrochemical potential $\mu_{\beta}$ of other electrodes by $I_{\alpha}=4 e^{2} / h \sum_{\beta} T_{\beta \alpha} \mu_{\alpha}-T_{\alpha \beta} \mu_{\beta}$ with $T_{\alpha \beta}$ the total transmission between the $\alpha$ and the $\beta$ electrodes [Figs. 1(c) and 1(d)]. The condition $I_{3}=0$ for a voltage probe gives $\mu_{3}=$ $\left(T_{31} \mu_{1}+T_{32} \mu_{2}\right) /\left(T_{31}+T_{32}\right)$. The transmission between electrodes 3 and 4 has been neglected since it corresponds to a second-order process. The potential of the voltage electrode $\mu_{3}$ can thus take any value between $\mu_{1}$ and $\mu_{2}$. Since the same holds for $\mu_{4}, R_{4 \mathrm{pt}}$ can be negative [see Figs. 1(c) and 1(d)]. Using $R_{2 \mathrm{pt}}=\left(\mu_{1}-\mu_{2}\right) / I$ and $R_{4 \mathrm{pt}}=\left(\mu_{3}-\mu_{4}\right) / I$, we find that $R_{4 \mathrm{pt}}$ takes any value between [10]

$$
-R_{2 \mathrm{pt}} \leq R_{4 \mathrm{pt}} \leq R_{2 \mathrm{pt}}
$$

The remarkable prediction of $R_{4 \mathrm{pt}}<0$ is difficult to observe experimentally. Some works on ballistic onedimensional conductors fabricated in semiconductors showed that $R_{4 \mathrm{pt}}$ can become slightly negative $[12,13]$. However, we believe that our data show for the first time significant negative $R_{4 \mathrm{pt}}$ that approaches $R_{2 \mathrm{pt}}$.

We have fabricated nanotube circuits with a new layout for four-point measurements. First, $\sim 1 \mathrm{~nm}$ diameter SWNTs grown by laser-ablation [14] or chemical-vapor deposition [15] are selected with atomic force microscopy (AFM). Voltage electrodes are then defined by positioning two MWNTs above the SWNT using AFM manipulation. We choose such voltage electrodes since the electric transmission between two crossed nanotubes is low [16,17]. Then, $\mathrm{Cr} / \mathrm{Au}$ electrodes are patterned for electric connection with standard electron-beam lithography techniques [see Fig. 1(a)]. Characteristics of the different devices are summarized in Table I. Measurements are always in the linear-transport regime.

We first use Coulomb blockade (CB) measurements to investigate the degree to which the MWNT probes are invasive. Nanofabricated electrodes have been reported to create barriers along SWNTs and to divide tubes in multiple quantum dots [7]. In those previous measurements most of the CB peaks are completely suppressed. Figure 2 shows a series of CB peaks that appear regularly when sweeping the gate voltage $V_{g}$ on the backgate. Peaks are at the same $V_{g}$ for measurements between different pairs of electrodes. This indicates that Au and MWNT electrodes probe the same quantum dot. Coulomb diamonds measurements (not shown) give that the charging energy $E_{c} \approx$ $5 \mathrm{meV}$. It has been shown that $E_{c} \approx \frac{5 \mathrm{meV}}{L[\mu \mathrm{m}]}$ for similarly prepared samples [18]. The dot length is thus $\sim 1 \mu \mathrm{m}$, which is consistent with the $600 \mathrm{~nm}$ separation between $\mathrm{Au}$ electrodes. These measurements suggest that MWNTs are sufficiently noninvasive to not divide the SWNT in multiple quantum dots.

The question of invasiveness can further be tested by measuring the length dependence of $R_{4 \mathrm{pt}}$. Two types of measurements have been performed. In Fig. 3(a), an AFM tip has been used to change the separation between two MWNTs. In Fig. 3(b), 6 MWNTs have been placed on a long SWNT, enabling the resistance measurement of multiple portions. $R_{4 \mathrm{pt}}$ linearly increases with the length, and $R_{4 \mathrm{pt}}$ tends to zero as the length is reduced to zero. A significant resistance contribution from the MWNTs would give a finite $R_{4 \mathrm{pt}}$ at zero length, in opposition to the measurements. This suggests that MWNTs are mainly noninvasive. Note, however, that measurements in Fig. 3(a) are rather scattered. This may come from the AFM manipulation that stretches the tube, deposits or removes some molecules adsorbed on the SWNT, or modifies the pressure applied by the MWNT on the SWNT. Thus, we cannot exclude a small resistance perturbation from the MWNTs and/or the AFM manipulation.

Our four-point measurement with no (or little) invasive voltage electrodes allows for the first direct measurement of the intrinsic resistance of a nanotube. The lowest resistance that we obtained is $1.5 \mathrm{k} \Omega$ for a $95 \mathrm{~nm}$ long section [Fig. 3(a)]. Such a low resistance is remarkable, since the resistance of quasi one-dimensional conductors is expected to be dramatically enhanced with the presence of disorder or phonons. This agrees with previous two-point measurements [19-22] since $R_{2 \mathrm{pt}}$ was shown to approach the quantum resistance $h / 4 e^{2}=6.5 \mathrm{k} \Omega$, which suggests low intrinsic resistance.

Further insight into transport properties is obtained by decreasing the temperature $T$. Figure 4 shows that $R_{4 \mathrm{pt}}$ does not change for $T$ above $\sim 80 \mathrm{~K}$, suggesting that the intrinsic resistance is related to some static disorder and not to phonons. At lower $T$, the resistance becomes larger and

TABLE I. Device characteristics at $300 \mathrm{~K}$. $L$ is the separation between the MWNTs and $L_{\mathrm{Au}-\mathrm{Au}}$ between the $\mathrm{Au}$ electrodes.

\begin{tabular}{lcccccc}
\hline \hline & $R_{4 \mathrm{pt}}(\mathrm{k} \Omega)$ & $L(\mathrm{~nm})$ & $l_{e}(\mathrm{~nm})$ & $L_{\mathrm{Au}-\mathrm{Au}}(\mu \mathrm{m})$ & & \\
\hline Device 1 & 1.5 & 95 & 408 & 2.7 & $\mathrm{SGS}^{\mathrm{a}}$ & CVD \\
Device 2 & 37.0 & 100 & 17 & 5.6 & $\mathrm{SC}^{\mathrm{b}}$ & LA \\
Device 3 & 2.7 & 150 & 358 & 1.0 & metal & CVD \\
Device 4 & 6.3 & 140 & 143 & 0.6 & metal & LA \\
Device 5 & 12.7 & 590 & 300 & 1.4 & metal & LA \\
\hline \hline
\end{tabular}

\footnotetext{
${ }^{a}$ Small-gap semiconductor with the current reduction occurring at $V_{g}>2 \mathrm{~V}$.

${ }^{b}$ Large-gap semiconductor with the threshold voltage at $\sim 40 \mathrm{~V}$. LA $=$ laser ablation. CVD $=$ chemical vapor deposition.
} 


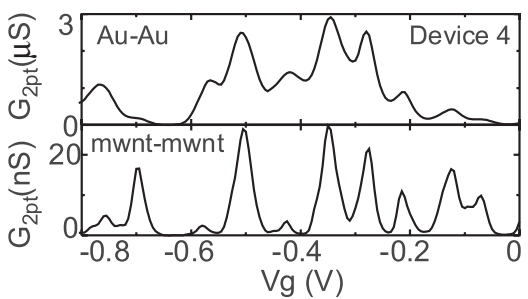

FIG. 2. Two-point conductance as a function of $V_{g}$ at $1.4 \mathrm{~K}$. $\mathrm{CB}$ peaks measured between different electrodes appear also at the same $V_{g}$ 's for Devices 1 and 3 .

starts to depend on the gate voltage. This will be discussed in more detail elsewhere.

Measurements above have shown that the four-point resistance is linear with length, in agreement with Ohm's law and Eq. (1) for a classical resistor. At $1.4 \mathrm{~K}$ this is, however, different, and $R_{4 \mathrm{pt}}$ can even become significantly negative. The inset of Fig. 5(a) shows that $R_{4 \mathrm{pt}}$ is $-29 \mathrm{M} \Omega$ near zero $V_{g}$. However, $R_{4 \mathrm{pt}}$ significantly drops to 0 for $V_{g}$ between -0.05 and $-0.1 \mathrm{~V}$ when the two-point conductance between Au electrodes increases, as shown in Fig. 2. This is in agreement with Eq. (2). Figs. 5(a) and 5(b) show that the modulations of $R_{4 \mathrm{pt}} / R_{2 \mathrm{pt}}$ are significant, with absolute values that reach as high as 0.6.

We now discuss the origin of the negative $R_{4 \mathrm{pt}}$. It might come from narrow diameter MWNTs or SWNT bundles $[23,24]$. The current pathway through different SWNTs may be complicated giving rise to negative $R_{4 \mathrm{pt}}$. However, such a classical effect should persist at higher $T$, which is not the case since $R_{4 \mathrm{pt}}>0$ at $T \geqslant 10 \mathrm{~K}$. Moreover, complicated current pathways give a finite nonlocal $R$ at high $T$ as recently shown [23]. We have not observed this for devices in this Letter.
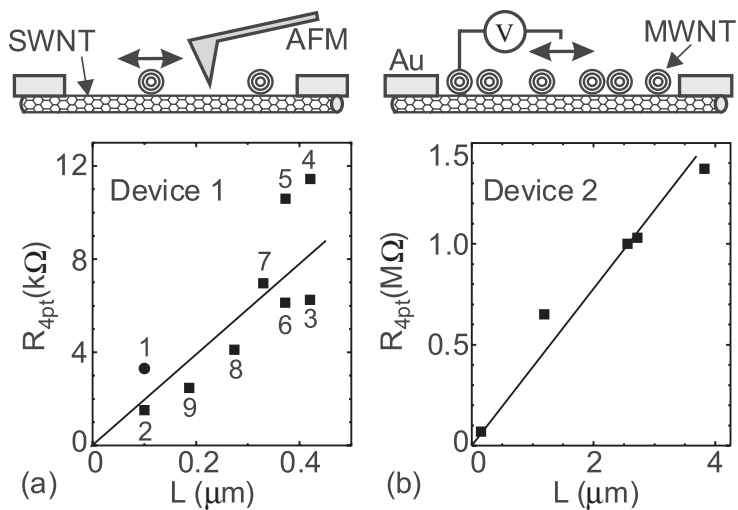

FIG. 3. Length dependence of $R_{4 \mathrm{pt}}$ at room temperature and $V_{g}=0$. (a) SWNT contacted by 2 MWNTs. One MWNT is displaced back and forth with an AFM tip. Points are numbered to describe the measurement sequence. Point 1 has been acquired one week before in the cryostat. Most points have been recorded while decreasing $L$, so that the $R$ enhancement with $L$ is not due to a structural degradation during the manipulation. (b) SWNT contacted by 6 MWNTs.

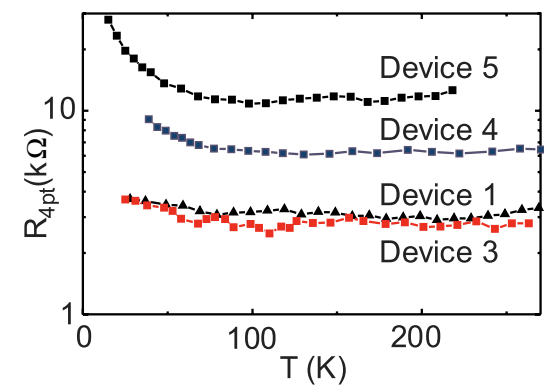

FIG. 4 (color online). Temperature dependence of $R_{4 \mathrm{pt}}$ measured at $V_{g}=0$.

The resistance ratio $R_{4 \mathrm{pt}} / R_{2 \mathrm{pt}}$ is given by [10]

$$
\frac{R_{4 \mathrm{pt}}}{R_{2 \mathrm{pt}}}=\frac{T_{31} T_{42}-T_{32} T_{41}}{\left(T_{31}+T_{32}\right)\left(T_{41}+T_{42}\right)} .
$$

Part of the fluctuations are expected to come from the Coulomb blockade observed at $1.4 \mathrm{~K}$ that leads to oscillations in $T_{i j}$ transmissions. However, regular $V_{g}=75 \mathrm{mV}$ CB oscillations cannot alone account for the rapid $V_{g} \sim$ $10 \mathrm{mV}$ fluctuations of $R_{4 \mathrm{pt}} / R_{2 \mathrm{pt}}$ in Fig. 5(a). We rather attribute those fluctuations to quantum-interference terms $[25,26]$ that are contained in $T_{i j}$ transmissions and that may arise from the superposition of different electronic paths between $i$ and $j$ electrodes [27]. Indeed, the disorder along the SWNT leads to different possibilities in the pathway between 2 electrodes [11]; see the inset of Fig. 5(b). It is also likely that the sign change originates from those interferences. Variations of $T_{i j}$ transmissions with $V_{g}$ are
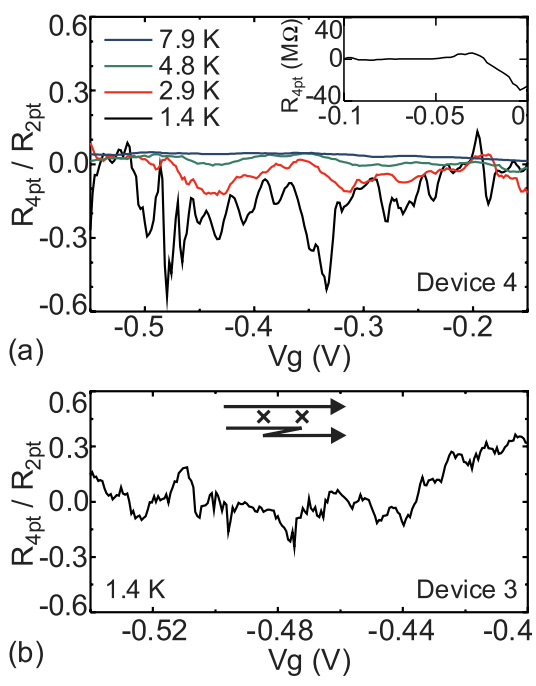

FIG. 5 (color online). Negative four-point resistance at low temperature. (a), (b) $R_{4 \mathrm{pt}} / R_{2 \mathrm{pt}}$ as a function of $V_{g}$. Similar results are also obtained for Device 1. $R_{2 \mathrm{pt}}$ measured between $\mathrm{Au}$ electrodes is checked to be lower than $R_{2 \mathrm{pt}}$ between MWNT electrodes. The inset of (a) shows $R_{4 \mathrm{pt}}\left(V_{g}\right)$ at $1.4 \mathrm{~K}$. $R_{4 \mathrm{pt}}$ is close to 0 for $V_{g}$ between -0.1 and $-0.6 \mathrm{~V}$. The inset of (b) shows two scattering centers that generate interference. 
then uncorrelated, enabling the sign reversal of the numerator in Eq. (3).

Note that the SWNT may have entered the regime of strong localization (SL) [11]. This is expected for SWNTs when the phase-coherence length is longer than a few times $l_{e}$. This may be the case since $l_{e}$ of Device 4 is $143 \mathrm{~nm}$ and negative $R_{4 \mathrm{pt}}$ suggests coherence over at least $L=$ $140 \mathrm{~nm}$. However, similar $R_{4 \mathrm{pt}}$ measurements are obtained for devices that are farther from the SL regime [Fig. 5(b), $\left.l_{e}=358 \mathrm{~nm}\right]$. Moreover, the two-point conductance modulation of Devices 1 and $3-5$ is quite regular when $V_{g}$ is swept (Fig. 2), in opposition to SL predictions [28]. We note that these devices are rather short (not much longer than $l_{e}$ ). This may explain that deviations from the diffusion regime due to SL are not significant.

So far, we have not taken into account the electronelectron interaction, which is expected to form a Luttinger liquid (LL) [17]. $R_{4 \mathrm{pt}}$ of a LL has not been calculated yet. However, interference due to impurities in a LL should also lead to negative $R_{4 \mathrm{pt}}$.

We have shown the transition of $R_{4 \mathrm{pt}}$ between the Ohm's law at $300 \mathrm{~K}$ and the quantum-interference regime at low temperature. The deviation from Ohm's law can become so dramatic that $R_{4 \mathrm{pt}}$ is negative. Hence it is likely that inclusion of these quantum-mechanical interference effects will ultimately be required in the design of practical multiterminal intramolecular devices.

We thank C. Delalande for support, L. Forro for MWNTs, and R. Smalley for laser-ablation SWNTs. The research in Paris has been supported by ACN, Sesame. Y.F.C. and M.S.F. acknowledge support from the U.S. National Science Foundation through Grant No. DMR0102950. LPA is CNRS-UMR8551 associated with Paris 6 and 7.

*Corresponding author. Email address: bachtold@1pa.ens.fr

[1] C. Joachim, J. K. Gimzewski, and A. Aviram, Nature (London) 408, 541 (2000).
[2] A. Bachtold et al., Phys. Rev. Lett. 84, 6082 (2000).

[3] P. J. de Pablo et al., Phys. Rev. Lett. 88, 036804 (2002).

[4] M. Freitag et al., Phys. Rev. Lett. 89, 216801 (2002).

[5] M. S. Gudiksen et al., Nature (London) 415, 617 (2002).

[6] Y. Yaish et al., Phys. Rev. Lett. 92, 046401 (2004).

[7] A. Bezryadin et al., Phys. Rev. Lett. 80, 4036 (1998).

[8] For example, a transmission of 0.4 at the interfaces generates an additional resistance of around $0.1 \mathrm{~h} / 4 \mathrm{e}^{2}$ by incoherently combining scattering matrices that describe backscattering along the tube and the coupling to the voltage electrodes [matrix (1) in M. Büttiker, Phys. Rev. B 32, R1846 (1985)].

[9] M. Büttiker, Phys. Rev. Lett. 57, 1761 (1986).

[10] M. Büttiker, IBM J. Res. Dev. 32, 317 (1988).

[11] S. Datta, Electronic Transport in Mesoscopic Systems (Cambridge University Press, Cambridge, 1997).

[12] Y. Takagaki et al., Solid State Commun. 68, 1051 (1988).

[13] R. de Picciotto et al., Nature (London) 411, 51 (2001).

[14] A. Thess et al., Science 273, 483 (1996).

[15] J. H. Hafner et al., J. Phys. Chem. B 105, 743 (2001).

[16] M. S. Fuhrer et al., Science 288, 494 (2000).

[17] B. Gao et al., Phys. Rev. Lett. 92, 216804 (2004).

[18] M. Bockrath et al., Science 275, 1922 (1997); J. Nygard et al., Appl. Phys. A 69, 297 (1999).

[19] W. Liang et al., Nature (London) 411, 665 (2001).

[20] J. Kong et al., Phys. Rev. Lett. 87, 106801 (2001).

[21] D. Mann et al., Nano Lett. 3, 1541 (2003).

[22] B. Babic and C. Schönenberger, Phys. Rev. B 70, 195408 (2004).

[23] B. Bourlon et al., Phys. Rev. Lett. 93, 176806 (2004).

[24] H. Stahl et al., Phys. Rev. Lett. 85, 5186 (2000).

[25] V. A. Gopar, M. Martinez, and P. A. Mello, Phys. Rev. B 50, 2502 (1994).

[26] M. Büttiker, Phys. Rev. B 40, R3409 (1989).

[27] Sweeping the magnetic field $B$ induces fluctuations of $R_{4 \mathrm{pt}}$ with the smallest fluctuations $\approx 6 \mathrm{~T}$ for Device 4 . The corresponding Zeeman energy, which is $0.7 \mathrm{meV}$ using the Landé factor $g=2$, is consistent with the rapid $V_{g} \approx 10 \mathrm{mV}$ fluctuations in Fig. 5(a) since the coupling efficiency measured from Coulomb diamonds is $\alpha=$ $1 / 15$.

[28] A. B. Fowler, A. Hartstein, and R. A. Webb, Phys. Rev. Lett. 48, 196 (1982). 